\title{
INTELLECTUAL DECISION SUPPORT IN CONTROL SYSTEM FOR THE PROCESS OF FORMING PROPERTIES OF MATERIALS WITH A COMPLEX INTERNAL STRUCTURE'
}

\author{
Korneev Andrey Mastislavovich, Buzina Olga Petrovna, Sukhanov Andrey Vladimirovich, Shipulin Ilya \\ Andreevich
}

\begin{abstract}
The lack of a fully developed theory and effective algorithms for system support for the process of optimal structuring of materials with complex structures (cast-iron alloys or discrete-reinforced materials) is currently actualizing the task of developing methods of intellectual support for decision-making in a management system that allows structuring materials that meet the criteria of operational reliability and efficiency on the material costs of their production. The purpose of this study is to improve the quality of materials with a complex internal structure based on models and algorithms for optimizing their structure and properties in the decision-making system using fuzzy control methods and stochastic searching on multidimensional spaces, and developing special mathematical, algorithmic and software for making effective management solutions. For this, the authors developed algorithms for making decisions on the formation of the structure and properties of an object of a complex structure, and also a system analysis of control models for the structuring of composite materials allowing efficient study of deformation processes inside objects whose geometric structure is complex (composite) and defined by random variable functions. The authors developed special software for the decision-making system for controlling the process of forming the structure and properties of materials, including the implementation of methods for mathematical modeling of the stress-strain state of the elements under study, stochastic methods for optimizing computational processes in the system, and methods for fuzzy control.
\end{abstract}

Keywords: complex internal structure, intellectual, control system

\section{INTRODUCTION}

Modern industrial technologies for the manufacture of materials with a complex composite internal structure based on discrete fibers differ in the multistage nature of the functioning processes, including the selection of physical and mechanical characteristics of composite materials, the choice of the internal geometric structure of discrete elements, and the prediction of reliability by specified criteria.

The absence of deterministic functional dependencies between the random variables of the processes involved in the formation of the material structure and the output parameters determining the composition and properties of the product necessitates the creation of a decision-making system based on optimization and control methods ensuring the obtaining of the required technological parameters for the construction of the structure satisfying the requirements reliability requirements.

An important step in improving the efficiency of such a system is the task of adapting and optimizing models, algorithms and computational methods aimed at systemic decision support at each stage of the production process.

The purpose of this research is to improve the quality of composite materials on the basis of models and algorithms for optimizing their structure and properties in the decision-making system using fuzzy control methods and stochastic search on multidimensional spaces, and the development of special mathematical, algorithmic and software for making effective management decisions. 
To achieve this goal, the following tasks are solved in the work: conducting a system analysis of models for managing the structuring of materials with a complex composite structure; the development of an algorithm for making decisions on the formation of the structure and properties of an object from a composite material; development of new and modification of existing algorithms of intellectual support of decision making on the basis of methods of random search and fuzzy control that allow calculating numerical parameters in the construction of the algorithm for stochastic search for the coefficients of characteristic diagrams in the structure control system for composite materials.

\section{RESEARCH METHOD}

Among the new research methodologies formed so far, four main and complementary approaches can be identified: synergistic, homeostatic, systemic and informational [1].

A systematic approach to the scientific knowledge of the properties and nature of the structure of materials with a composite structure gave a powerful impetus to the development in science of new directions characterized by the application of known methods of system theory to the development of control algorithms for the formation of rational chemical compositions for structures in various industries. An important methodological and scientific value of the system approach is that it allows researchers to identify and formulate the principles of systemic nature, manifested in the process of formation of chemical compounds. In modern system methodologies used in the application to the development and structuring of materials with a composite structure, an approach is increasingly being used that is a set of methods and tools that allow one to investigate both the properties, structure and functions of individual elements and composites of an object, interaction, that is, to study the intrastructural processes as a whole, presenting them as systems with all the complex interelement links by the interaction of elements on the system itself and on the surrounding first medium $[1,2,3,4]$.

When studying any complex system, object or phenomenon, the system approach is based on their holistic vision, with the main feature of the system approach being the prevailing role of the whole over the individual and complex over simple $[5,6]$. Note that although the main properties and results of the system's activity depend to a large extent on the composition and properties of the elements entering into it, but in principle can not be studied at the level of exclusively the characteristics of these elements [4, 6, 7]. This observation was taken into account by the researchers using the system approach in the application to the design and calculation of materials having a composite structure $[2,3,4,6]$. For technical objects with a complex internal structure, it is the holistic, synthetic description that makes it possible to link their various elements, previously considered separately, together $[8,9]$.

One can also not ignore the processes of controlling the structure formation of materials with a complex composite structure occurring in the system [8]; these processes require a separate study of the system with respect to the information circulating in it.

Since in the modern understanding the system is a collection of interconnected elements united by functional integrity and unity of purpose (here the property of the whole system is not reduced to the sum of the properties of its individual elements), its properties as a whole are set not only by the properties of the individual elements but also properties of its structure. For most types of systems that allow to structure a material with certain properties, among the significant components of the structure of the control system indicate:

- the spatial arrangement of all elements of the system;

- stable links between the elements of the system;

- the internal arrangement of the system; 
- laws that determine the mutual influence, the interaction of elements within the system.

Since any system is formed as a result of the interaction of its constituent elements, which gives the system new properties that are not characteristic of individual elements, the number of properties of the system, as a rule, exceeds the sum of the properties of the individual elements; the difference is a new system properties. Completeness of the internal structure of the system is characterized by functional integrity, and several interrelated elements of the structure form a system only if the relations between the elements of the system generate a new system quality different from the previous ones, called integrative.

Strict, correct and unambiguous definition of the system for today does not exist. However, there are many different, albeit close to each other, definitions of the system and its structure. More often define a system as a set of elements, united through a series of relationships that generate a systemic, or integrative, quality by which the given set is distinguished from the environment, and to which each of its components is attached.

In some cases, the concept of the system is used incorrectly, for example, in cases where the integrative quality does not take place (the unification of parts in the system does not lead to the appearance of a new property different from those that the individual parts had before unification).

An example of such a system is iron-carbon, whose structure consists of various components (austenite, cementite, graphite, perlite) with certain properties (specific heat, coefficient of casting expansion, thermal conductivity, etc.). It is obvious that the composition and structure of pig iron depend on the constituent components.

Taking into account, it should be noted that it would be erroneous, as a system, to consider the formal union of individual components in (the properties of the system here are identical to the sum of the properties of the given layers) $[2,3]$.

The most significant properties and features of the system are indicated in [5]. The system here refers to the totality of elements that are in a definite connection with each other, and form the integrity and unity. The most widespread and recognized this definition was obtained from domestic researchers $[2,3]$.

Some types of objects that are called systems are not covered by the currently available system definitions, but this does not prevent distinguishing systems from other objects $[2,3,5]$. The most characteristic features of systems contained in the definitions are the following: the presence of a structure; integrity of the set of system elements; stable links between the elements of the system; the organization of developing systems (Figure 1). 


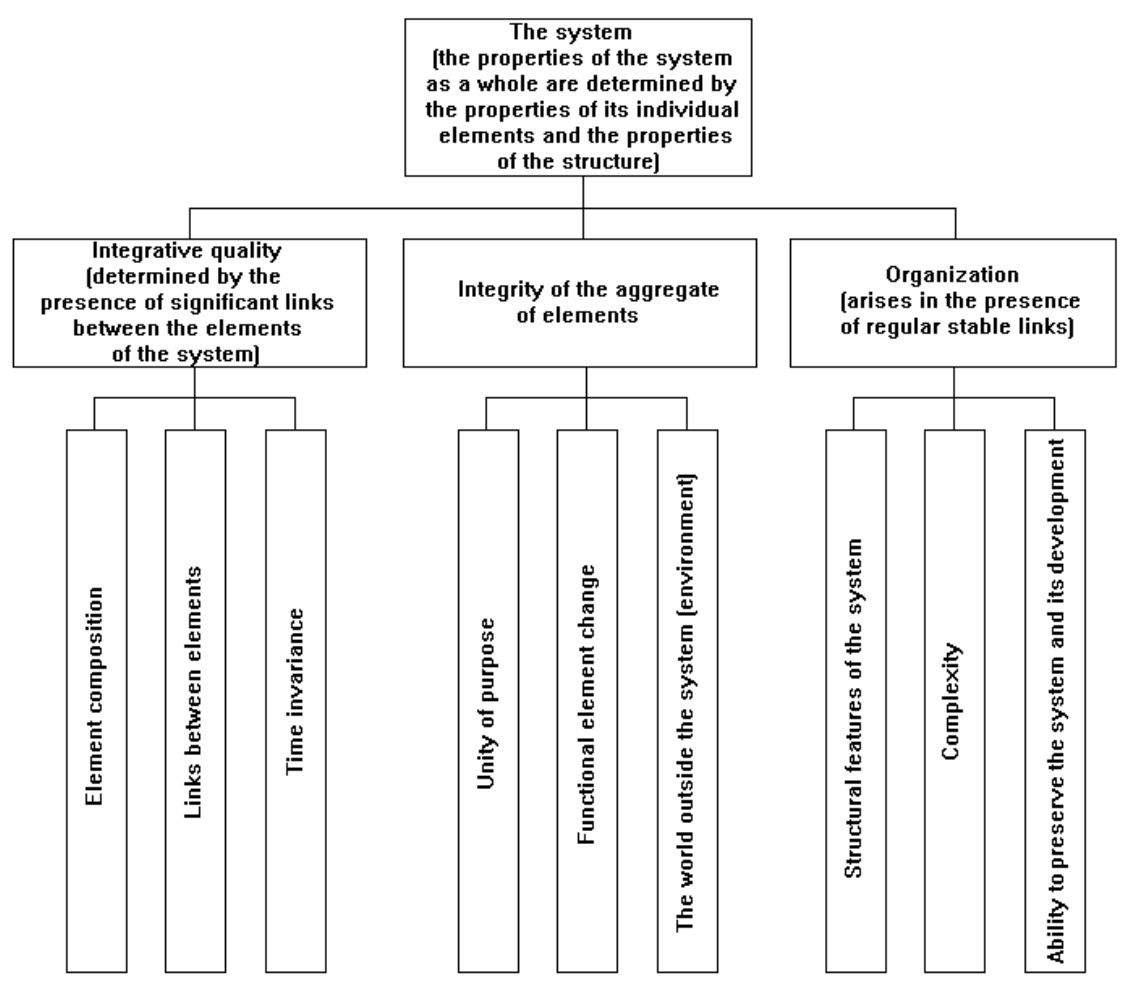

Fig.1. Characteristic features of the system

The structure is a form of representation of the object in the form of separate parts, as well as a set of relationships that are only possible between elements within the system itself. The system is also that which remains unchanged when the state of the system changes and when the system performs certain operations [15]. It is always necessary to indicate which part of the system and what its properties and attributes are structural and which are not, since the structure is part of the system. In practice, many real systems do not lend themselves to a simple description because of the high complexity of their structures. In this connection, the structural elements of the system were understood as its smallest part, the behavior of which is still subject to the laws of the system. Their own structural elements can be contained within the elements of the structure themselves, which generates the hierarchy principle in the construction of connections between systems, which presupposes subordination of the subsystem to the system and the system of the supersystem. Such systems are called structured systems [2, 3].

In the study of such systems, under the rule, in most cases, one often understands the often observed property (dependence or connection) that is inherent in several objects, phenomena and processes, established as a result of the experiment (the process of forming physical characteristics, the dependence of the operator's control on the information parameters) [10].

\section{RESULTS AND ANALYSIS}

For materials with a complex discrete structure, an algorithm for making decisions on the formation of its optimal structure and properties has been developed (Figure 2). 
The Turkish Online Journal of Design, Art and Communication - TOJDAC

ISSN: 2146-5193, March 2018 Special Edition, p. 353-362

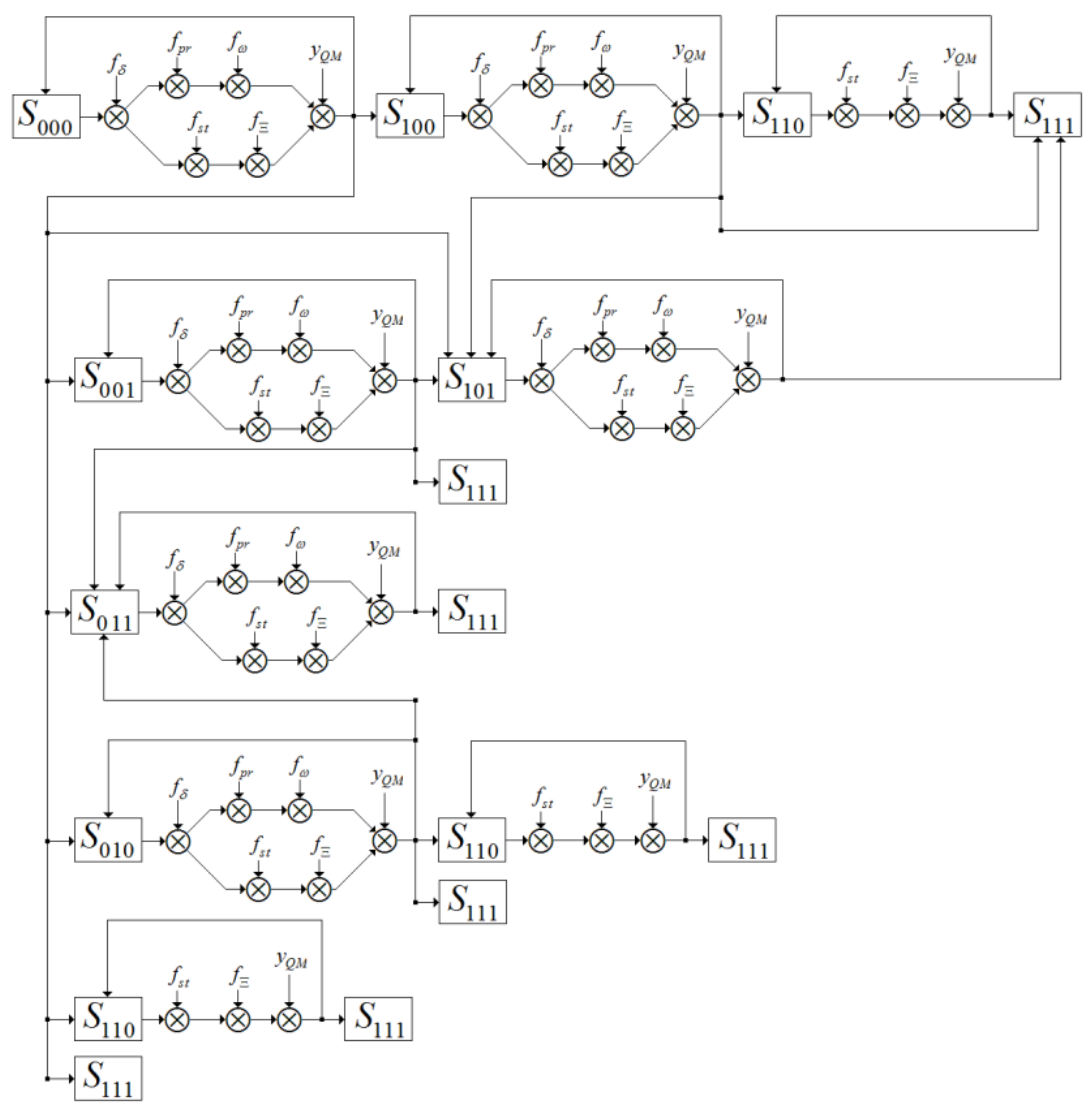

Fig. 2. Scheme of the decision-making system for forming the properties of materials with a discrete internal structure 


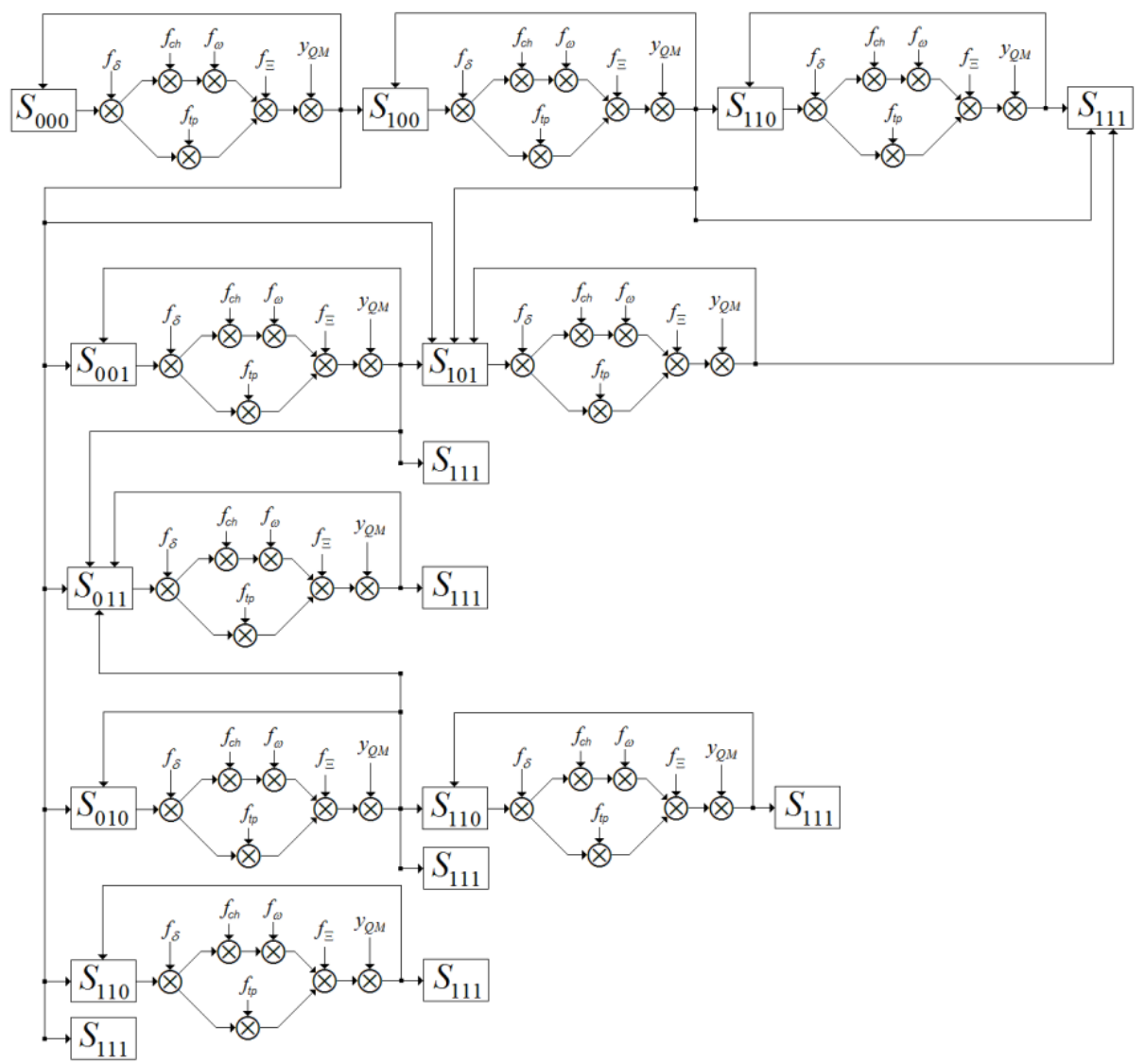

\section{Fig. 3. Scheme of the decision-making system for the formation of the structure and chemical composition of the cast iron alloy}

The scheme of the decision-making system for the formation of the optimal composition for the material of a disperse structure (cast iron alloy) with the application of functionals is shown in Figure 3.

In the structure of the system, based on the selected system-forming properties, the following decisionmaking functionals are defined that contain algorithmic algorithms and mathematical models that allow optimizing the properties and structure of materials:

- $f_{\delta}$ - the decision-making functional about the method of changing the structure of a composite material using the multicriterion optimization method based on the specified quality criteria;

- $\mathrm{f}_{\mathrm{pr}}$ - the decision-making functional function for the quantitative measure of hardening of matrix material;

- $\mathrm{f}_{\omega}$ is the functional determining optimal parameters of the curvature of the deformation diagram for a matrix of known strength;

- $\mathrm{f}_{\mathrm{st}}$ - the decision-making functional about the quantitative measure of changing the structure of disperse reinforcement;

- $\mathrm{f}_{\Xi}$ is the functional that determines the optimal parameters for the numerical coefficients of the deformation diagrams; 
- $\mathrm{y}_{\mathrm{QM}}$ is a functional that simulates external effects on composite material and determines the degree of reliability by the given criteria (determines the state of the decision-making system).

The states in which a system can be located are characterized by a list of reliability criteria that an element of a composite material satisfies or does not satisfy. The list of possible states of the system is presented in Table 1.

The system can go from one state to another, but the final state must be as close as possible to $\mathrm{S}_{111}$, or ideally the system must reach this state.

Table 1. Possible states of the system

\begin{tabular}{|c|c|c|c|}
\hline \multirow{2}{*}{$\begin{array}{l}\text { State designation } \\
\text { systems }\end{array}$} & \multicolumn{3}{|c|}{$\begin{array}{l}\text { Criteria for the reliability of an element from } \\
\text { composite material }\end{array}$} \\
\hline & strength & deflection & crack resistance \\
\hline$S_{000}$ & not done & not done & not done \\
\hline$S_{001}$ & not done & not done & fulfilled \\
\hline$S_{010}$ & not done & fulfilled & not done \\
\hline$S_{011}$ & not done & fulfilled & fulfilled \\
\hline$S_{100}$ & fulfilled & not done & not done \\
\hline$S_{101}$ & fulfilled & not done & fulfilled \\
\hline$S_{110}$ & fulfilled & fulfilled & not done \\
\hline$S_{111}$ & fulfilled & fulfilled & fulfilled \\
\hline
\end{tabular}

Analysis of optimization methods based on meta-heuristic algorithms of stochastic global minimum search showed that for modeling optimal values of numerical coefficients of deformation diagrams of objects of complex disperse structure, modeling using several schemes and with a choice of modifications is necessary.

Based on the results of the analysis, the following schemes of optimization algorithms are chosen: the Boltzmann scheme of modifications A, B and C (Annealing), the Cauchy scheme of modifications A, B and C (Annealing), ultrafast search (Very Fast Annealing) and the algorithm Xin Yao. These schemes are the basis for the work of the functionals $\mathrm{f}_{\omega}$ and $\mathrm{f}_{\Xi}$ of the decision-making system $[11,12]$.

The modified stochastic search algorithm based on the Boltzmann scheme contains the Gibbs distribution function, which is modified by applying the parameter Imod (the coefficient of variability of the distribution function):

$$
I_{\mathrm{mod}}=\left|1-\sqrt[n]{\prod_{j=1}^{n}\left|\frac{M_{x j}^{\prime}}{M_{x j}^{e}}\right|^{1 / j}}\right|
$$


This coefficient takes into account the accumulation of "deviations" in the values of $M_{x j}$ from the values of the actual experiment $M_{x j}^{e}$. The role of the modification coefficient is that the function $E$ should pass to a new state $E^{`}$ of the system with a higher probability in the case when the values of $M_{x j}$ and $M_{x j}^{e}$ approach.

As an instrument of intellectual support in choosing the numerical parameters of the algorithms for optimizing the coefficients of the characteristic diagrams, the method of non-precise control of Takagi and Sugeno is applied in the developed decision-making system. This method is used here to determine the coefficient $\chi$ that affects the rate of convergence of the search algorithm:

- for the Boltzmann scheme: $T(k)=\frac{T_{0}}{\ln \left(k^{\chi}\right)}$,

- for the Cauchy scheme: $T(k)=\frac{T_{0}}{\sqrt[4]{k^{\chi}}}$.

To select the optimal vector from the set of alternative solutions, and also to reduce the initial set of alternatives, the Pareto method is chosen at the initial stage of the problem solution. The principle of optimality is used to optimize solutions and find a compromise between the requirements for decisions, which are determined by particular criteria.

In the system of decision-making on the structuring of composite materials, the problem of finding a compromise among the following efficiency criteria is solved:

1. The strength of the composite material (concrete) matrix under compression, $y_{1}=R_{b}(M P a)$.

2. Compressive strength of the composite material, $\mathrm{y}_{2}=\mathrm{R}_{\mathrm{fb}}(\mathrm{MPa})$.

3. Initial modulus of elasticity of composite material, $\mathrm{y}_{3}=\mathrm{E}_{\mathrm{fb}}(\mathrm{MPa})$.

4. Frost resistance, $\mathrm{y}_{4}$ (cycles).

5. Water-resistant, y5 (MPa).

6. Material costs of production, $\mathrm{y}_{6}$.

For example, for a material such as fibro concrete, the vector of possible solutions to the multicriteria problem of optimizing the structure and properties of composite materials contains the following parameters: $\mathrm{x}_{1}$ - cement-water ratio; $\mathrm{x}_{2}$ - normal density of cement paste, $\% ; \mathrm{x}_{3}$ - workability on a conventional scale; $\mathrm{x}_{4}$ - duration of heat treatment, $\mathrm{h} ; \mathrm{x}_{5}$ - content of fiber reinforcement, $\mathrm{kg} ; \mathrm{x}_{6}$ - type of discrete fibers; $\mathrm{x}_{7}$ - the resulted expenses for cement, $\mathrm{kg}^{-1} ; \mathrm{x}_{8}$ - the resulted expenses for the additive, rub / $\mathrm{kg} ; \mathrm{x}_{9}$ - the resulted expenses for a fiber, $\mathrm{kg}^{-1} ; \mathrm{x}_{10}$ - content of the additive, $\mathrm{kg} ; \mathrm{x}_{11}$ - activity of cement, MPa.

To each vector $X=\left\{x_{1}, x_{2}, x_{3}, x_{4}, x_{5}, x_{6}, x_{7}, x_{8}, x_{9}, x_{10}, x_{11}\right\}$ from the space of alternatives R11 there corresponds a vector of values of the criteria

$$
Y(X)=\left[f_{1}(X), f_{2}(X), f_{3}(X), f_{4}(X), f_{5}(X), f_{6}(X)\right] .
$$

Comparing the two alternatives $X^{\prime \prime}, X^{\prime} \in D$, we verify the fulfillment of the inequality $f_{i}\left(X^{\prime}\right) \geq f_{i}\left(X^{\prime \prime}\right)$ for all $i=1, \ldots, 6$. If these inequalities are satisfied and at least one inequality is strict, then we choose the 
alternative $X^{\prime}$ as a solution more efficient than following the Pareto optimality principle. We note here that for the function $f_{6}\left(x_{1}, x_{2}, x_{3}, x_{4}, x_{5}, x_{7}, x_{8}, x_{9}\right)$ there must be an inequality $f_{6}\left(X^{\prime}\right) \leq f_{6}\left(X^{\prime \prime}\right)$, since the material costs $y_{6}$ are minimized. If from the set of alternative solutions for some point $X^{0} \in D$ we do not find a more efficient one $X^{0}$, then we take the point as the Pareto-optimal solution of the multicriteria problem.

In more detail, the vector of alternative solutions is represented as:

$$
Y(X)=\left(\begin{array}{l}
f_{1}\left(x_{1}, x_{2}, x_{3}, x_{4}, x_{10}, x_{11}\right) \\
f_{2}\left(x_{1}, x_{2}, x_{3}, x_{4}, x_{5}, x_{6}, x_{10}, x_{11}\right) \\
f_{3}\left(x_{1}, x_{2}, x_{3}, x_{4}, x_{5}, x_{6}, x_{10}, x_{11}\right) \\
f_{4}\left(x_{1}, x_{2}, x_{3}, x_{4}, x_{10}, x_{11}\right) \\
f_{5}\left(x_{1}, x_{2}, x_{3}, x_{4}, x_{10}, x_{11}\right) \\
f_{6}\left(x_{1}, x_{2}, x_{3}, x_{4}, x_{5}, x_{7}, x_{8}, x_{9}\right)
\end{array}\right),
$$

and the problem of optimizing the structure and composition of composite materials using the Pareto method is formulated as follows:

$$
Y(X)=\left\{X: \arg \max _{X \in D}\left[\left(\sum_{i=1}^{5} f_{i}(X)\right)-f_{6}(X)\right]\right\} .
$$

\section{CONCLUSION}

Based on the proposed schemes An automated system designed to make efficient decisions in the design of the composition and structure of composite materials containing discrete fibers has been developed. The system also allows you to predict the properties of the final product, - bent elements from the formed material. The user interface allows you to enter the following parameters: initial geometric characteristics of the element under study; data on the initial values of the strength and deformation characteristics of the composite material matrix; parameters of the disperse distribution of discrete fibers in the matrix; the required values of the quality and reliability criteria.

Thus, the structure of the special mathematical and software of the decision making system for the synthesis of composite materials, including the implementation of methods of fuzzy control and multicriteria optimization, the feature of which is the possibility of using weight coefficients for the structuring of a material with specified properties, differing the universality of its application, and allowing to formulate the optimal composition and structure of materials, taking into account the requirements for the criterion of operational reliability and efficiency of material costs

\section{REFERENCES}

Danilov A.M. Systems and models [Text]: Textbook /A.M. Danilov. - Penza: PGAAS, 1995, - 200 p. Garkina, I.A. Experience in optimizing a multipurpose system / IA Garkina, TN. Volkova // Modern scientific research and innovations. - 2014. - №10. - P.1.

Garkina IA, Danilov AM, Smirnov VA Flocculation in disperse systems of control system and information technology. - 2008. - No. 2.3 (32) .- P. 344-346.

Prangishvili I.V. Improving the management efficiency of complex organizational and socio-economic systems / I.V. Prangishvili // Problems of Management. - 2005. - №5. - P.28-32. 
Blauberg I.V. Formation and essence of the system approach: monograph / I.V. Blauberg, E.G. Yudin. Moscow: Nauka, 1973. - 270 p.

Prangishvili I.V. A systematic approach and system-wide regularities: a scientific publication / I.V. Prangishvili - M .: SYNTHEG, - 2000. - 528 p.

Sadovsky, V.N. Investigations on the general theory of systems. Sadovsky, E.G. Yudin. - M .. Progress. 1969. - $520 \mathrm{p}$.

Buslenko N.P. Modeling of complex systems: monograph / N.P. Buslenko. - Moscow: Nauka, - 1968. $355 \mathrm{p}$.

Kukhtenko A.R. The main tasks of managing complex systems / A.R. Kukhtenko // Complex control systems: Sat. sci. works. - Kiev. - 1968. - Issue. 1. - P.27-39.

Sorokin DS, Danilov AM Regularities in composites from positions of system analysis // Young scientist. 2014. - №10. - P. 206-208.

Ingber L. Adaptive simulated annealing (ASA): Lessons learned // Journal Control and Cybernetics. 1995.

Ingber L. Very fast simulated re-annealing // Mathematical and Computer Modelling. 12. 1989. P. 967973. 